E-Mail: q.vanest@rathenau.nl

Internet: http://www.rathenau.nl

Drs. Lucien Hanssen, Director

Deining Consultancy for Social Communication

Peter Scheerstraat 26, NL-6525 DE Nijmegen

The Netherlands

E-Mail: deining@wxs.nl

\section{Szenarien einer liberalisierten Stromversorgung}

\author{
von Georg Förster, Fachhochschule Nürtin- \\ gen, und Wolfgang Weimer-Jehle, Akade- \\ mie für Technikfolgenabschätzung in Ba- \\ den-Württemberg
}

Der vorliegende Beitrag beschreibt in komprimierter Form die Vorgehensweise und einige prägnante Ergebnisse des 2001 an der Akademie für Technikfolgenabschätzung in Baden-Württemberg durchgeführten Projekts „Szenarien einer liberalisierten Stromversorgung". Als spezielle diskursive Methode zur Erstellung möglicher Szenarien für den deutschen Strommarkt im Jahr 2010 wurde das Cross-Impact-Verfahren eingesetzt. Mit dieser Szenario-Technik, die in ihren Grundzügen beschrieben wird, lassen sich die Wechselwirkungen der wesentlichen Systemgrößen des Strommarktes untereinander analysieren. Die Szenarien wurden in Zusammenarbeit mit Experten aus zehn wissenschaftlichen Einrichtungen und unter Einbindung relevanter Akteure unterschiedlicher Interessensgruppen entwickelt.

\section{Einleitung}

Durch die Binnenmarktrichtlinie für Strom hat die Europäische Union die Voraussetzungen für die Öffnung der Elektrizitätsmärkte im europäischen Binnenmarkt geschaffen. Somit ist die Versorgung dieses leitungsgebundenen Energieträgers unter Wettbewerbsbedingungen gestellt. Seit dem Inkrafttreten der Richtlinie befindet sich die Stromwirtschaft in einem dynamischen Umbruch, der mit erheblichen Veränderungen verbunden ist. Teilweise handelt es sich hierbei um Übergangserscheinungen, teilweise werden die entstandenen Neuerungen auch in Zukunft bestehen bleiben. Mit der grundlegenden Reform des Ordnungsrahmens für die europäische Stromwirtschaft verändern sich die Marktstrukturen, die relevanten Akteure, die Strompreise, die angebotenen Produkte, das Nachfrageverhalten und nicht zuletzt die Einflussmöglichkeiten und Aufgaben des Staates und der Kommunen.

Der Wettbewerb und die Lenkung von Angebot und Nachfrage über den Markt sollen dazu beitragen, die Effizienz der Stromversorgung zu verbessern. Dies führt zu völlig neuen 
Handlungsfeldern bei den relevanten Akteuren. Auf der Seite der Energieversorger steht die Steigerung der Wettbewerbsfähigkeit mit allen ihren Konsequenzen im Vordergrund. Aber auch für den Kunden ergeben sich durch die freie Wahl des Stromversorgers neue Möglichkeiten. Letztendlich ist auch der Staat trotz der Liberalisierung gefordert, da die Stromversorgung im Interesse der Allgemeinheit liegt. Er muss die notwendigen Rahmenbedingungen schaffen, damit die Märkte dazu befähigt werden, die Ziele der Versorgungssicherheit, der Preiswürdigkeit und der Umweltverträglichkeit innerhalb des Wettbewerbs zu erreichen.

In der momentanen Übergangsphase vom monopolistischen Markt zum voll ausgebildeten Wettbewerb sind einerseits viele Veränderungen sichtbar geworden, andererseits besteht noch in vielen Bereichen eine Unsicherheit, wie sich diese in Zukunft entwickeln werden bzw. welche Tendenzen sich noch ausbilden. Für Politik, Wirtschaft und Gesellschaft ist es daher wünschenswert, die sich bereits abzeichnenden Entwicklungen so früh wie möglich zu erkennen, um sich rechtzeitig auf diese einstellen zu können. Die Akademie für Technikfolgenabschätzung in Baden-Württemberg (TA-Akademie) hat daher mit dem Projekt ,Szenarien einer liberalisierten Stromversorgung" dieses aktuelle Thema aufgegriffen, um entsprechend ihrem Auftrag zur Klärung der Zusammenhänge in diesem Themenbereich einen Beitrag zu leisten. Das Ziel hierbei war es vor allem, konsistente Szenarien, die mögliche Zustände im Jahr 2010 beschreiben, herauszuarbeiten. Diese Zeitangabe ist mit einer Unschärfe von 1 bis 2 Jahren zu sehen. Da sich aber gerade in den Jahren nach 2010 erhebliche Veränderungen (z.B. im Kraftwerkspark) ergeben werden, können die getroffenen Aussagen nur sehr eingeschränkt über diesen Zeitraum hinaus extrapoliert werden. Das Jahr 2010 wurde deshalb als Zeitbezugspunkt für die Szenarien gewählt, da man davon ausgehen kann, dass bis dahin die momentan noch andauernde Übergangsphase abgeschlossen ist und sich eine vollständige Liberalisierung mit einem gänzlich funktionierenden und diskriminierungsfreien Wettbewerb eingestellt hat. Ein weiteres Argument für das Jahr 2010 als Prognosehorizont war die Tatsache, dass bei Szenarien, die einen späteren Zeitpunkt als Bezug haben, die Unschärfe bzw. die Streuweite der Aussagen zwangsläufig größer wird.

\section{Projektverlauf}

Eine Aufarbeitung der möglichen Konsequenzen der Strommarkt-Liberaliserung muss in den Blick nehmen, dass im politischen Bereich, bei den Wirtschaftsakteuren, aber auch in der Wissenschaft zu vielen Fragen der Energieversorgung erhebliche Unterschiede in den Einschätzungen und Zielvorstellungen bestehen. Um die Entwicklung einer einseitigen Sichtweise zu verhindern, wurde die Bearbeitung der Problemstellung als strukturierter Expertendiskurs angelegt. Das Projekt wurde überwiegend aus Eigenmitteln der TA-Akademie finanziert. Dadurch konnte möglichen Vermutungen einer interessensgebundenen Projektführung von vorne herein die Grundlage entzogen werden. Das Projekt bestand aus folgenden Elementen:

1. In einer Pilotstudie der TA-Akademie wurde der Stand der politischen und fachlichen Diskussion aufgearbeitet, die wichtigsten Fragestellungen eingegrenzt und relevante Akteure und Experten mit ihrer spezifischen Verortung in der Kontroverse identifiziert (Förster 2001).

2. In Vorgesprächen mit Wissenschaftlern und Vertretern von Ministerien, Stromerzeugern und Stromverbrauchern wurden die Ergebnisse der Pilotstudie, die Zielsetzung des Projektes und die Informationsbedürfnisse der Gesprächspartner diskutiert.

3. Mit einem Projektkreis, der sich aus neun externen Wissenschaftlern (vgl. Kasten) zusammensetzte, wurden in drei Workshops verschiedene qualitative Szenarien einer liberalisierten Stromversorgung für Deutschland im Jahr 2010 entwickelt. Das methodische Verfahren zur Szenarienkonstruktion wird in Abschnitt 3 näher beschrieben, die wichtigsten Ergebnisse sind in Abschnitt 4 dargestellt.

4. Die zuvor für Deutschland skizzenhaft entwickelten Zukunftsbilder wurden in ihrer Entsprechung für Baden-Württemberg durch Modellrechnungen des Instituts für Energiewirtschaft und rationelle Energieanwendung der Universität Stuttgart regionalisiert und quantifiziert. Dieser Projektteil 
wurde durch das Ministerium für Umwelt und Verkehr Baden-Württemberg gefördert.

\section{Teilnehmer an den Expertenworkshops}

\section{Externe Experten}

- Dr. H. Bradke, Fraunhofer-Institut für Systemtechnik und Innovationsforschung, Karlsruhe

- Dipl.-Wirtsch.-Ing. W. Bräuer, Zentrum für Europäische Wirtschaftsforschung, Mannheim

- Dr. U. Fahl, Institut für Energiewirtschaft und Rationelle Energieanwendung, Stuttgart

- Prof. Dr. U. Leprich, Institut für ZukunftsEnergieSysteme, Saarbrücken

- Dr. R. Menges, Energiestiftung SchleswigHolstein, Kiel

- Dr. J. Nitsch, Deutsches Zentrum für Luft- und Raumfahrt (Institut für Technische Thermodynamik, Abt. Systemanalyse und Technikbewertung), Stuttgart

- Prof. Dr.W. Pfaffenberger, Bremer Energieinstitut, Bremen

- Prof. Dr.W. Schulz, Energiewirtschaftliches Institut an der Universität Köln

- Dipl.-Phys. S. Thomas, Wuppertal Institut, Wuppertal

- Dipl.-Ing. C. Timpe, Öko-Institut, Freiburg

\section{TA-Akademie}

- Dr. G. Förster

- Dr. D. Schade

- Dr.W.Weimer-Jehle

Der Szenarioprozess wurde durch einen Projektbeirat, der sich aus Vertretern unterschiedlicher Interessengruppen (große Energieversorger, Stadtwerke, Umweltschutzverbände, Verbraucherverbände, Ministerien etc.) zusammensetzte, begleitet. Die Aufgabe des Projektbeirates war es, zusätzliche Sichtweisen, Argumente und Informationen in den Prozess mit einzubringen sowie die Arbeitsergebnisse zu kommentieren.

\section{Methodisches Vorgehen zur Szenario- konstruktion}

Aufgabe des Expertenkreises war die diskursive Erstellung von Grobszenarien einer liberalisierten Stromversorgung Deutschlands im Jahr 2010. Durch die verschiedenen Szenarien sollte der Raum der möglichen Entwicklungen abgesteckt werden. Für die diskursive Erstellung anstatt der gebräuchlicheren modellgestützten Analyse sprach, dass auf diese Weise eine Viel- falt von Sichtweisen eingebracht und auch ,weiche" Einsichten in die Systemzusammenhänge, Marktprozesse und deren Wechselwirkung mit politischen Eingriffen verwertet werden konnten. Der damit verbundene Verzicht auf ein vollquantitatives Bild erschien uns angesichts der Vorteile des Ansatzes akzeptabel, insbesondere da die Unsicherheit von Zukunftseinschätzungen den Wert von quantitativen Details im vorliegenden Problem ohnehin relativieren.

Zur Strukturierung der diskursiven Szenarioentwicklung wählten wir mit dem Cross-ImpactVerfahren (CI-Verfahren) ein Standardverfahren der Szenariotechnik. Es wurde vor etwa 35 Jahren zur groben Erfassung von systemaren Wechselwirkungen entwickelt (Gordon, Hayward 1968). Seither ist es in vielen Variationen besonders im Bereich der strategischen Planung in Gebrauch und wurde von uns für den Einsatz in diesem Projekt mit den Zielrichtungen Verfahrenstransparenz und Diskursfähigkeit weiterentwickelt. Die Methode beruht auf einem Systemverständnis von Systemgrößen, die in Form von fördernden oder hemmenden Einflüssen wechselseitig wirken (Abb. 1). 
Der Raum der grundsätzlich möglichen Systemzustände (,Szenarien“) wird durch die Vielfalt der Zustandskombinationen der Matrix repräsentiert. Bei der einfachen Beispielmatrix in Abb. 1 sind dies lediglich $3 \times 3$ Kombinationen, in praktischen Anwendungsfällen sind die Matrizen selbstverständlich größer und die Anzahl der Kombinationsmöglichkeiten kann Tausende oder Millionen von Szenarien beinhalten. Die meisten dieser kombinatorisch erzeugbaren Szenarien sind fachlich unsinnig. Formal drückt sich dies in einer durch Auszählung ermittelbaren Inkonsistenz in den Wirkungsbilanzen der CI-Matrix aus. Auf diese Weise kann bei Vorliegen einer CI-Matrix rechnergestützt eine vollständige Durchmusterung der kombinatorischen Szenario-Vielfalt vorgenommenen werden und die i.A. geringe Anzahl von Szenarien aussortiert werden, die mit den formulierten Wechselwirkungen in Einklang stehen. Durch die vollständige Durchmusterung des kombinatorischen Raums besteht die Chance, auch unerwartete Szenarien zu thematisieren, die abseits der übli- das Verfahren vorgeschlagenen Szenarien. Eine detaillierte Beschreibung des angewendeten Verfahrens sowie seiner Vor- und Nachteile ist im Projekt-Endbericht (Förster 2002) enthalten. Inzwischen wurde die von der Akademie entwickelte Verfahrensvariante auch in anderen Projekten angewendet (siehe Förster, WeimerJehle 2003). Die Anwendung des Verfahrens innerhalb dieses Projektes erfolgte innerhalb folgender Stufen:

1. In einem vorbereiteten Arbeitsschritt wurden alle Experten um eine Einschätzung gebeten, welche politisch-wirtschaftlich-energietechnischen Systemgrößen relevant für die aufgeworfene Fragestellung sind. Mit einer Einflussanalyse wurden die genannten Größen nach den Kategorien der Abb. 1 (Rahmengrößen, aktive Systemgrößen, passive Systemgrößen) geordnet. In einem ersten Workshop wurden die wichtigsten energiewirtschaftlichen und energiepolitischen Rahmenannahmen vereinbart, die allen weiteren Überlegungen zugrunde gelegt

\section{Abb. 1: Das Cross-Impact-Verfahren*}

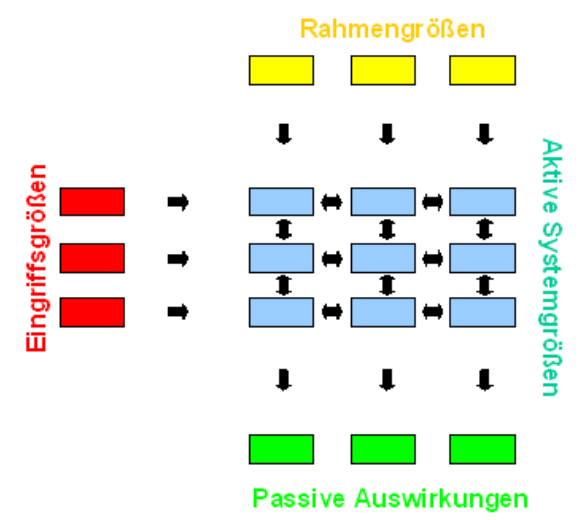

\begin{tabular}{|c|c|c|}
\hline $\begin{array}{l}\text { Wechsel- } \\
\text { wirkungen }\end{array}$ & $\begin{array}{c}A \\
\text { a1 a2 a3 }\end{array}$ & $\begin{array}{c}\text { B } \\
\text { b1 b2 b3 }\end{array}$ \\
\hline $\begin{array}{ll}\text { A } & \text { a1 } \\
& \text { a2 } \\
& \text { a3 }\end{array}$ & & $\begin{array}{rrr}-1 & 0 & 1 \\
0 & 0 & 0 \\
2 & 0 & -2 \\
\end{array}$ \\
\hline $\begin{array}{ll}\text { B } & \text { b1 } \\
\text { b2 } \\
\text { b3 }\end{array}$ & $\begin{array}{rrr}2 & -1 & -1 \\
1 & -2 & 1 \\
-2 & -1 & 3\end{array}$ & \\
\hline
\end{tabular}

* Das CI-Verfahren versteht ein System als Komplex wechselwirkender Größen (links). Die Einwirkungsmöglichkeiten der Größen aufeinander sind durch Pfeile angedeutet. Die CI-Matrix (rechts für den Fall von zwei Systemgrößen A und B mit jeweils 3 möglichen Zuständen) formuliert das System durch die Einschätzung des hemmenden oder fördernden Charakters der Wechselwirkung i.A. durch eine diskrete Stärkenskala (z.B.+3 für starke Förderung, -3 für starke Hemmung).

chen Denkschemen eine gewisse Plausibilität aufweisen und die damit die Diskussion bereichern können. Auf der anderen Seite erfordert die Unvollständigkeit der CI-Systembeschreibung eine qualifizierte Nachprüfung der durch werden sollten. Weiterhin wurde vereinbart, welche Systemgrößen in die Analyse aufgenommen werden und durch welche Zustände sie beschrieben werden sollen. Das Ergebnis dieser Problemstrukturierung wird in 
Abschnitt 4 beschrieben und führte auf eine Cross-Impact-Struktur mit insgesamt 15 Größen (davon 11 aktiv und 4 passiv). Der Raum der möglichen Entwicklungen war damit kombinatorisch durch 73.728 formal mögliche Szenarien erschlossen.

2. Jeder Experte formulierte sein Systemverständnis, indem er eine Cross-Impact-Matrix für diese Struktur ausfüllte. Die TA-Akademie wertete alle Matrizen getrennt aus und bestimmte in jedem Fall die Szenarien, die mit dem jeweiligen Wechselwirkungsbild konsistent waren. Daraus ergab sich eine Liste von 36 Szenarien. Diese Szenarienschar beinhaltete die Meinungsheterogenität des Expertenkreises, da es für die Aufnahme in diese Liste nicht erforderlich war, dass sich ein Szenario aus mehreren oder sogar allen Experten-Matrizen ergeben musste. Auch Szenarien, die auf lediglich eine ExpertenMatrix zurückgingen und bei anderen Experten-Matrizen als inkonsistent gelten müssten, wurden hier berücksichtigt, um gegebenenfalls auch Einzeleinschätzungen als Diskussionsvorschläge zu erhalten.

3. Zur Reduktion des Diskussionsaufwandes wurden die 36 Szenarien nach Ähnlichkeitsgesichtspunkten gruppiert. Es ergaben sich vier Szenario-Gruppen, aus denen je ein Repräsentant gewählt wurde. Diese Gruppierungs- und Auswahlverfahren erfolgten rechnergestützt nach offengelegten, rein formalen Regeln und beinhalteten bis zu diesem Zeitpunkt keine subjektiven Auswahlschritte.

4. Auf der Basis dieser vier, aus dem formalen Analyseverfahren als Vorschlag hervorgegangenen Szenarien wurde in einem weiteren Expertenworkshop eine fachliche Diskussion durchgeführt. Die Szenarien wurden entsprechend ihren Wesenszügen mit Überschriften versehen - ein interpretativer Akt, der interessanterweise das Gegenteil des üblichen Verfahrens der Szenarioanalyse darstellt, in dem von Szenario-Leitbildern ausgegangen wird und die Konstruktion der Szenarien den vorgegebenen Leitbildern folgt. Die vorgeschlagenen Szenarien wurden inhaltlich von den Experten reflektiert und in wenigen Punkten modifiziert. Die so entstandenen Szenarien sind Gegenstand des nächsten Abschnitts.

\section{Ergebnisse}

Gemäß dem methodischen Vorgehen aus Abschnitt 3 wurde aus jeder Szenarien-Gruppe jeweils ein repräsentatives Szenario ausgewählt. Diese vier Szenarien $\left(\mathrm{S}_{1}-\mathrm{S}_{4}\right)$, die das Spektrum der wahrscheinlichen Entwicklungen bis zum Jahr 2010 abdecken, setzen sich aus zwei Teilen (Rahmen- und Systemgrößen) zusammen. Der erste Teil beinhaltet eine Reihe von Rahmengrößen (siehe Abb. 1), die für alle vier Szenarien gleich sind. Hierbei handelt es sich um Entwicklungen bzw. Zustände für das Jahr 2010, die von allen am Projekt beteiligten Experten in ihrer Eintrittswahrscheinlichkeit als sehr hoch eingeschätzt wurden. Der zweite Teil besteht aus einem Satz von Systemgrößen mit verschiedenen Ausprägungen (siehe Abb. 1 ,aktive Systemgrößen“ und ,passive Auswirkungen“), in denen sich die vier Szenarien unterscheiden.

An dieser Stelle sollen nur zwei wichtige Rahmengrößen exemplarisch aufgezeigt werden. Eine Grundvoraussetzung für alle Szenarien war beispielsweise die Annahme, dass die bisher eingeschlagene Richtung der Politik zum Klimaschutz und zur Ressourcenschonung weiter verfolgt und ausgeweitet wird. Auf Grund dessen beinhalten alle vier Szenarien zusätzliche staatliche Eingriffe im Bereich der umweltorientierten Gesetzgebung, die in unterschiedlicher Art und Weise über die bisherigen Maßnahmen hinausgehen (siehe weiter unten). Eine weitere Rahmengröße war die Hypothese, dass die Weltmarktpreise für Rohöl bis zum Jahr 2010 real (d.h. inflationsbereinigt) nur geringfügig $(5-10 \%)$ in der trendgemäßen Entwicklung ansteigen werden. Kurzfristige starke Schwankungen, die von diesem Trend abweichen, sind selbstverständlich möglich. Eine Reihe weiterer Rahmengrößen aus den Bereichen „Staat", „Energieträger", ,Stromnet-

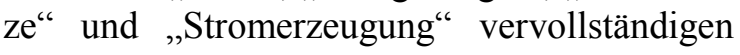
die Basis für die Szenarien.

Entsprechend der in der Einleitung angesprochenen Sektoren, in denen sich Veränderungen ergeben, lassen sich die in ihren Ausprägungen variierenden Systemgrößen (= zweiter Teil der Szenarien) in drei Gruppen einteilen:

1. „staatliche Eingriffe“

(Gesetzgebung für regenerative Energieerzeugung und Kraft-Wärme-Kopplung (KWK) sowie zur Reduktion der $\mathrm{CO}_{2}-$ 
Emissionen, Steueranteil an den Strompreisen, Energiepolitik mit Wirkung auf die Stromeffizienz)

2. "Stromversorgung“"

(Strompreisentwicklung, Stromimport-Saldo, $\mathrm{CO}_{2}$-Emissionen durch die Stromerzeugung, Gas-Anteil am Energieträgermix, Kraftwerkspark (Anteil der dezentralen Anlagen), Anbieterstruktur (große/lokale/internationale Anbieter etc.), Angebotsstruktur (z. B. Energiedienstleistungen etc.), Beschäftigung in der Stromwirtschaft)

3. „Verbraucherverhalten“

(Einsparverhalten im privaten Bereich, freiwillige private Ökostromnachfrage, Stromeffizienzsteigerung im Bereich der Industrie)

Aus den oben stehenden Systemgrößen, deren unterschiedliche Ausprägungen an dieser Stelle nicht dargestellt sind, und ihren von den Experten kodierten Wechselwirkungen ließen sich konsistente Kombinationen (Szenarien) finden. Innerhalb der vier ausgewählten Szenarien wird für den ersten Bereich (,staatliche Eingriffe“) deutlich, dass sich weiterreichende Maßnahmen zur Erhöhung der Stromerzeugung aus regenerativen Energiequellen und KWK sowie zur Verringerung der $\mathrm{CO}_{2}$-Emissionen durchgesetzt haben werden. Sie unterscheiden sich jedoch in den Wegen, auf denen dies erreicht wird. Entweder können die Förderungen der Stromerzeugung aus regenerativer Energie und der KWK sowie die $\mathrm{CO}_{2}$-Reduktionsmaßnahmen EU-weit harmonisiert werden oder diese Umsetzung findet mittels nationaler Instrumente statt. Weitere wesentliche Unterschiede innerhalb der vier Szenarien ergaben sich im dritten Bereich („Verbraucherverhalten“). Hier kann es innerhalb des eigenständigen ökologischen Marktagierens entweder bei dem derzeitigen Trend (geringe Effizienzanstrengungen und geringe Ökostromnachfrage) bleiben oder aber es kommt zu verstärkten Stromeffizienzanstrengungen auf der privaten bzw. der industriellen Seite sowie einer erhöhten ÖkostromNachfrage. Ausgehend von diesen Unterschieden wurden die vier Szenarien folgendermaßen benannt:

- Szenario $S_{1}$ : „Europäische Harmonisierung und geringes ökologisch orientiertes Marktagieren auf der Verbraucherseite“
- Szenario $\mathrm{S}_{2}$ : „Europäische Harmonisierung und ökologisch orientiertes Marktagieren durch verstärktes privates Umwelthandeln“

- Szenario $S_{3}$ : „Nationale Instrumente und zusätzliche staatliche Maßnahmen im Bereich der Stromeffizienz"1

- Szenario $\mathrm{S}_{4}$ : „Nationale Instrumente und starkes ökologisch orientiertes Marktagieren auf der Verbraucherseite“"

Auf Grund der weitreichenden energiepolitischen Annahmen ergeben sich im zweiten Bereich („Stromversorgung") bei einigen Systemgrößen trendgemäß ähnliche Entwicklungen. So steigen in allen vier Szenarien die Strompreise inflationsbereinigt um mehr als $10 \%$ und der Importstrom-Saldo nimmt auf ca. 5\% der Bruttostromerzeugung zu. Des Weiteren sinken die $\mathrm{CO}_{2}$-Emissionen aus der Stromerzeugung um mehr als $10 \%$ gegenüber dem Jahr 2000 . Hierzu tragen der prozentual zunehmende Anteil von Gas am Energieträgermix" sowie der steigende Anteil dezentraler Anlagen am Kraftwerkspark (regenerative Stromerzeugungs- sowie KWK-Anlagen) wesentlich bei. Weiterreichende statistische Auswertungen des CIVerfahrens ergaben, dass es sich hierbei um sehr starke Trends mit einer hohen Eintretenswahrscheinlichkeit handelt. Über die Entwicklungen der Anbieterstruktur, der Vermarktung des Produktes Strom sowie der Beschäftigung in der Stromwirtschaft lassen sich keine so eindeutigen Parallelen innerhalb der Szenarien finden.

Bei der Spezifizierung der vier „Deutschland-Szenarien" auf Baden-Württemberg wurde deutlich, dass sich diese weitgehend auf BadenWürttemberg übertragen lassen. Lediglich im Bereich der Stromnachfrage im Wärmemarkt kam es teilweise zu Abweichungen. Der Wert dieser zusätzlichen Ausarbeitung liegt darin, dass im Vergleich zu den mehr qualitativen Aussagen der „Deutschland-Szenarien“ speziell für Baden-Württemberg auch quantitative Ergebnisse vorliegen.

Eine vollständige Aufführung und Beschreibung der Rahmengrößen sowie der Systemgrößen und der kompletten Szenarien findet sich in (Förster 2002); ebenso die quantitativen Resultate der Modellrechnungen für BadenWürttemberg.

Eine zentrale Aussage der Szenarien ist, dass zwar durch die Liberalisierung und die daraus folgenden Reaktionen der Marktakteure 
der Strommarkt grundlegend verändert wurde, die entscheidenden richtungsweisenden Impulse in der Hauptsache jedoch nach wie vor von den staatlichen Eingriffen in das System ausgehen. Obwohl sich der Staat mit der Liberalisierung einerseits aus seiner Verantwortung zurückgezogen hat, sind andererseits bestimmte Maßnahmen und Eingriffe erforderlich, damit er seine energiepolitischen Ziele hinsichtlich Klimaschutz und Umweltverträglichkeit innerhalb des neu geschaffenen Wettbewerbs durchsetzen kann.

\section{Anmerkung}

1) Bei Szenario $S_{3}$ entwickelt sich ein ökologisches Marktagieren auf der Verbraucherseite in Form von verstärktem Einsparverhalten im privaten Bereich sowie forcierten Effizienzsteigerungen im Bereich der Industrie.

\section{Literatur}

Förster, G., 2001: Szenarien einer liberalisierten Stromversorgung - Pilotstudie. Arbeitsbericht Nr. 180 der Akademie für Technikfolgenabschätzung in Baden-Württemberg, Stuttgart. Pdf-Datei: http://www.ta-akademie.de

Förster, G., 2002: Szenarien einer liberalisierten Stromversorgung - Endbericht. Analysen der Akademie für Technikfolgenabschätzung in BadenWürttemberg, Stuttgart. Pdf-Datei: http://www.taakademie.de

Förster, G., Weimer-Jehle, W., 2003: CrossImpact-Methode. In: Umwelt- und Klimaschutz in liberalisierten Energiemärkten - Die Rolle erneuerbarer Energieträger. Forum für Energiemodelle und Energiewirtschaftliche Systemanalysen in Deutschland (Hrsg.). Heidelberg: Physica-Verlag (in Vorbereitung)

T.J. Gordon, H. Hayward, 1968: Initial experiments with the cross-impact matrix of forecasting. Futures, Vol. 1, No. 2, S. 100-116

\section{Kontakt}

Dr. Wolfgang Weimer-Jehle

Akademie für Technikfolgenabschätzung in BadenWürttemberg

Industriestr. 5, 70565 Stuttgart

Tel.: +49 (0) $711 / 9063$ - 104

Fax: +49 (0) $711 / 9063$ - 299

E-Mail: wolfgang.weimer-jehle@ta-akademie.de Internet: http://www.ta-akademie.de
Prof. Dr. Georg Förster

Fachhochschule Nürtingen

Außenstelle Geislingen an der Steige Parkstr. 4, 73312 Geislingen a. d. St. Tel.: +49 (0) $7331 / 22$ - 559

Fax: +49 (0) $7331 / 22$ - 560

E-Mail: foerster@asg.fh-nuertingen.de Internet: http://www.fh-nuertingen.de 